\title{
Two-colour mid-infrared absorption in an InAs/GaSb-based type II and broken-gap quantum well
}

\author{
X F Wei ${ }^{1}$, W Xu ${ }^{1,2}$ and Z Zeng ${ }^{1}$ \\ ${ }^{1}$ Key Laboratory of Materials Physics, Institute of Solid State Physics, Chinese Academy of \\ Sciences, Hefei 230031, People's Republic of China \\ ${ }^{2}$ Department of Theoretical Physics, Research School of Physical Sciences and Engineering, \\ Australian National University, Canberra ACT 0200, Australia \\ E-mail: wen105@rsphysse.anu.edu.au
}

Received 15 July 2007, in final form 27 September 2007

Published 19 November 2007

Online at stacks.iop.org/JPhysCM/19/506209

\begin{abstract}
We examine contributions from different transition channels to optical absorption in an InAs/GaSb-based type II and broken-gap quantum well (QW). In such a structure, because both electron and hole subbands are occupied by the conducting carriers, new channels open up for electronic transition via intra- and inter-layer scattering mechanisms. We find that two absorption peaks can be observed through inter-subband transitions within the same material layer. The absorption induced by the inter-layer transition is rather weak due to a small overlap of electron and hole wavefunctions. The results suggest that InAs/GaSb-based type II and broken-gap QWs can be employed as twocolour photodetectors working at mid-infrared bandwidth at relatively high temperatures up to room-temperature.
\end{abstract}

\section{Introduction}

Optical absorption or transmission experiments are one of the main tools used for characterizing and studying semiconductor-based quantum well (QW) structures, particularly their energy levels, without involving the excitonic effects [1]. In a conventional QW, there are mainly two mechanisms responsible for direct optical absorption induced by electronic transition excited by the radiation field. One is to excite electrons in the valence-band across the forbidden zone into the unoccupied electron subbands in the conduction-band, namely the inter-band transition. Another is to excite electrons or holes from occupied subbands to unoccupied subbands in the conduction-band or valence-band in an n-type or p-type QW, that is the intersubband transition. In III-V based QWs, the former process requires much larger photon energy than the latter one due to a large energy gap between the conduction- and valence-bands. 


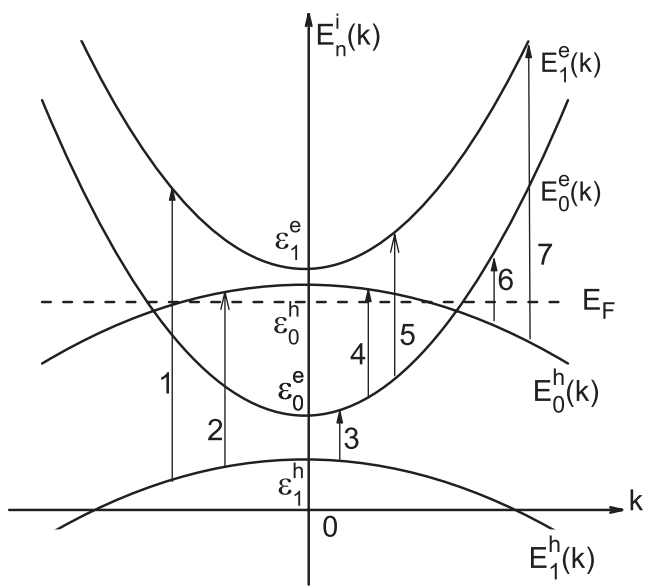

Figure 1. Schematic diagram of the optical absorption channels (1-7 as indicated) in a type II and broken-gap QW structure. Here, $E_{n}^{j}(k)=\varepsilon_{n}^{j}+(-1)^{j} \hbar^{2} k^{2} / 2 m_{j}^{*}$ is the energy spectrum for an electron $(j=e=2)$ or a hole $(j=h=1), \varepsilon_{n}^{j}$ is the $n$th subband energy for an electron or a hole, and $E_{\mathrm{F}}$ is the Fermi energy. Note that channels 1, 3, 4, 6 and 7 correspond to inter-band or inter-layer transitions and channels 2 and 5 are inter-subband transition channels for, respectively, holes in the GaSb layer and electrons in the InAs layer.

In sharp contrast, in a type II and broken-gap QW structure ${ }^{3,4}$, because both electron- and hole-subbands in different material layers are occupied by carriers, new channels open up for electronic transition accompanied by the absorption of photons. As indicated in figure 1, when only the two lowest electron-subbands and the two highest hole-subbands are considered and only the ground electron-subband and ground hole-subband are populated by the conducting carriers, there are seven possible transition channels for optical absorption scattering. In such a case, direct optical absorption scattering can occur through exciting electrons and holes in the occupied subbands into unoccupied subbands (channels 1, 2, 4, 5, 6 and 7 in figure 1). As a result, multi-coloured optical absorption can be observed due to different transition energies for electrons and holes in different material layers. Interestingly, owing to the overlap of the electron and hole wavefunctions at the interface of the InAs/GaSb heterojunction, interband or inter-layer electronic transitions accompanied by optical absorption (channels 1, 4, 6 and 7 for transitions from occupied subbands to unoccupied subbands and channel 3 for transition within occupied subbands in figure 1) are also possible. In contrast to the inter-band optical transition in a conventional QW, inter-band transition in a type II QW corresponds to the charge transfer between different material layers which can cause photoconduction along the growth direction [4]. Similarly to a conventional QW, inter-subband transitions within the same well layer (channel 5 for electrons and channel 2 for holes in figure 1) are also the important channels for optical absorption. In a type II and broken-gap QW, because both electron and hole subbands are occupied by the conducting carriers, inter-subband optical absorption can be achieved through interactions of the radiation fields with both electrons and holes in different well layers. Hence, from a viewpoint of basic physics and device applications, it is of great importance and significance to examine how these optical transition events contribute to the overall absorption in an type II and broken-gap QW, and this has become one of the prime motivations of this theoretical study.

3 Proposed theoretically by Altarelli [2].

4 Realized experimentally first by Munekata et al [3]. 


\section{Theoretical considerations and approaches}

It is known both experimentally [5] and theoretically [6] that in strained InAs/GaSb QWs, the hybridization of the electron and hole dispersions along the 2D-plane can occur. This hybridization can result in a mini-gap at the anti-crossing point between the electron and hole dispersion relations. For a type II QW with a narrow width of the $\operatorname{InAs}$ layer $\left(L_{\mathrm{InAs}} \leqslant 10 \mathrm{~nm}\right)$ and a thick width of the GaSb layer $\left(L_{\mathrm{GaSb}} \geqslant 10 \mathrm{~nm}\right)$, the mini-gap is found to be about several $\mathrm{meV}[5,6]$. However, it is found theoretically [6] that with increasing InAs layer thickness (i.e. $L_{\mathrm{InAs}} \geqslant 15 \mathrm{~nm}$ and $L_{\mathrm{GaSb}}=10 \mathrm{~nm}$ ), the hybridization mini-gap decreases rapidly. Very recent experimental results [7] also indicate that for an InAs/GaSb-based type II and brokengap QW with a relatively wide InAs well width $\left(L_{\mathrm{InAs}}=17 \mathrm{~nm}\right)$ and a relatively narrow GaSb well width $\left(L_{\mathrm{GaSb}}=5 \mathrm{~nm}\right)$, the electron and hole densities, along with even the quantum mobilities for electrons and holes, can be determined simply through measuring the Shubnikovde Haas oscillations. The measured electron and hole densities agree with those obtained from theoretical calculations in which the hybridization effect is not included [7, 8]. This implies that for an InAs/GaSb QW with a thick InAs width and a narrow GaSb width, the effect of the hybridization of the electron and hole dispersions can be largely reduced. Moreover, in this study we focus our attention on the consequences of the presence of mid-infrared radiation fields which are polarized linearly along the growth-direction of the QW. In such a case, due to the fact that electron/hole-photon interactions do not vary the electron/hole momentum along the 2D-plane, the presence of the hybridization of the dispersion relations in the 2D-plane does not affect significantly the optical transition in the mid-infrared bandwidth at which the photon energy $\hbar \omega$ is much larger than the hybridization mini-gap. Hence, in the present study, we consider an InAs/GaSb QW with a thick InAs layer width and a narrow GaSb layer width along with a high radiation frequency so that the effect of the hybridization of the electron and hole dispersions can be neglected.

Here we employ the semi-classic Boltzmann equation as the governing transport equation to study the response of the carriers (electrons and holes) in a type II QW to the applied radiation field. For an electron $(i=\mathrm{e})$ or a hole $(i=\mathrm{h})$, we have

$$
\frac{\partial f_{n}^{i}(\mathbf{k}, t)}{\partial t}=g_{\mathrm{s}} \sum_{j, \mathbf{k}^{\prime}, n^{\prime}}\left[F_{n^{\prime} n}^{j i}\left(\mathbf{k}^{\prime}, \mathbf{k}, t\right)-F_{n n^{\prime}}^{i j}\left(\mathbf{k}, \mathbf{k}^{\prime}, t\right)\right] .
$$

Here, $F_{n n^{\prime}}^{i j}\left(\mathbf{k}, \mathbf{k}^{\prime}, t\right)=f_{n}^{i}(\mathbf{k}, t)\left[1-f_{n^{\prime}}^{j}\left(\mathbf{k}^{\prime}, t\right)\right] W_{n n^{\prime}}^{i j}\left(\mathbf{k}, \mathbf{k}^{\prime}\right), n$ is the electronic subband index, $\mathbf{k}=\left(k_{x}, k_{y}\right)$ is the electron or hole wavevector along the 2D-plane, $f_{n}^{i}(\mathbf{k}, t)$ is the momentumdistribution functions for an electron or a hole at a state $|\mathbf{k}, n\rangle, g_{\mathrm{s}}=2$ counts for spin degeneracy and $W_{n n^{\prime}}^{i j}\left(\mathbf{k}, \mathbf{k}^{\prime}\right)$ is the steady-state electronic transition rate for scattering of an electron or a hole from a state $|\mathbf{k}, n\rangle$ in layer $i$ to a state $\left|\mathbf{k}^{\prime}, n^{\prime}\right\rangle$ in layer $j$. In equation (1), the effect of the EM field has been included within the time-dependent electron/hole distribution functions and within the electronic transition rate. Thus, to avoid double counting, the force term induced by the EM field does not appear on the left-hand side of the Boltzmann equation. For cases where the electronic transition is induced by electron or hole interactions with the radiation field, the transition rate can be obtained by Fermi's golden rule, which reads $W_{n n^{\prime}}^{i j}\left(\mathbf{k}, \mathbf{k}^{\prime}\right)=W_{n n^{\prime}}^{i j}(\mathbf{k}) \delta_{\mathbf{k}^{\prime}, \mathbf{k}}$ and

$$
W_{n n^{\prime}}^{i j}(\mathbf{k})=\frac{2 \pi}{\hbar}\left(\frac{e \hbar F_{0}}{m_{i}^{*} \omega}\right)^{2}\left|X_{n n^{\prime}}^{i j}\right|^{2} \delta\left[E_{n}^{i}(\mathbf{k})-E_{n^{\prime}}^{j}(\mathbf{k})+\hbar \omega\right],
$$

where $F_{0}$ and $\omega$ are, respectively, the electric field strength and frequency of the EM field, $X_{n n^{\prime}}^{i j}=\int \mathrm{d} z \psi_{n^{\prime}}^{j *}(z) \mathrm{d} \psi_{n}^{i}(z) / \mathrm{d} z, E_{n}^{\mathrm{e}}(\mathbf{k})=\hbar^{2} k^{2} / 2 m_{\mathrm{e}}^{*}+\varepsilon_{n}^{\mathrm{e}}$ and $E_{n}^{\mathrm{h}}(\mathbf{k})=-\hbar^{2} k^{2} / 2 m_{\mathrm{h}}^{*}+\varepsilon_{n}^{\mathrm{h}}$ are, respectively, the energy spectra for an electron and a hole with $m_{j}^{*}$ being the effective mass for 
an electron or a hole, and $\psi_{n}^{i}(z)$ and $\varepsilon_{n}^{i}$ are, respectively, the wavefunction and subband energy for an electron or a hole along the growth direction.

It is known that there is no simple and analytical solution to equation (1) with the electronic transition rate given by equation (2). In this work, we apply the usual balance-equation approach to solve the problem [9]. For the first moment, the energy-balance equation [10] can be derived by multiplying both sides of equation (1) by $g_{\mathrm{s}} \sum_{\mathbf{k}} E_{n}^{i}(\mathbf{k})$. In doing so, we obtain two energy-balance equations respectively for an electron and a hole and, from them, the total electronic energy transfer rate due to electron/hole interactions with photons is obtained as

$$
P=\mathcal{P}_{\mathrm{e}}+\mathcal{P}_{\mathrm{h}}=\sum_{i, j} P_{i j}
$$

where $\mathcal{P}_{i}=g_{\mathrm{s}} \partial\left[\sum_{n} \sum_{\mathbf{k}} E_{n}^{i}(\mathbf{k}) f_{n}^{i}(\mathbf{k}, t)\right] / \partial t$ is the electronic energy transfer rate for electrons or holes and

$$
P_{i j}=4 \hbar \omega \sum_{n^{\prime}, n, \mathbf{k}} f\left(E_{n}^{i}(\mathbf{k})\right)\left[1-f\left(E_{n^{\prime}}^{j}(\mathbf{k})\right)\right] W_{n n^{\prime}}^{i j}(\mathbf{k}) .
$$

Here we have used a statistical energy distribution such as the Fermi-Dirac function as the electron/hole distribution function at a steady-state. Namely, we have taken $f_{n}^{i}(\mathbf{k}, t) \simeq$ $f\left(E_{n}^{i}(\mathbf{k})\right)$ with $f(x)=\left[1+\mathrm{e}^{\left(x-E_{\mathrm{F}}\right) / k_{\mathrm{B}} T}\right]^{-1}$ and $E_{\mathrm{F}}$ being the Fermi energy (or chemical potential). The optical absorption coefficient induced by electron and hole interactions with the EM field can be calculated through [11]

$$
\alpha=\alpha_{0}\left(2 \hbar P / e^{2} F_{0}^{2}\right)=\sum_{i, j} \alpha_{i j},
$$

where $\alpha_{0}=e^{2} /\left(\hbar \sqrt{\kappa} \epsilon_{0} C\right), \kappa$ and $\epsilon_{0}$ are respectively the dielectric constants of the material and the free space, and $C$ is the velocity of the light in vacuum. For optical absorption induced by electronic transition within the same well layer (i.e. within the InAs layer for electrons and within the GaSb layer for holes)

$$
\alpha_{i i}=\beta_{i} \frac{k_{\mathrm{B}} T}{\pi} \sum_{n^{\prime}, n} \frac{\left|X_{n n^{\prime}}^{i i}\right|^{2} B_{n^{\prime} n}^{i} \Gamma_{i}}{\left(\varepsilon_{n^{\prime}}^{i}-\varepsilon_{n}^{i}-\hbar \omega\right)^{2}+\Gamma_{i}^{2}} .
$$

For optical absorption induced by electronic transition between different well layers, we have

$$
\alpha_{\mathrm{eh}}=\beta_{\mathrm{e}}\left(M^{*} / m_{\mathrm{e}}^{*}\right) \sum_{n^{\prime}, n} \Theta\left(\varepsilon_{n^{\prime}}^{\mathrm{h}}-\varepsilon_{n}^{\mathrm{e}}-\hbar \omega\right)\left|X_{n n^{\prime}}^{\mathrm{eh}}\right|^{2} F_{n n^{\prime}}^{\mathrm{he}}
$$

for transition from the InAs layer to the GaSb layer and

$$
\alpha_{\mathrm{he}}=\beta_{\mathrm{h}}\left(M^{*} / m_{\mathrm{h}}^{*}\right) \sum_{n^{\prime}, n} \Theta\left(\varepsilon_{n^{\prime}}^{\mathrm{h}}-\varepsilon_{n}^{\mathrm{e}}+\hbar \omega\right)\left|X_{n^{\prime} n}^{\mathrm{he}}\right|^{2} F_{n n^{\prime}}^{\mathrm{eh}}
$$

for transition from the GaSb layer to the InAs layer. Here, $\beta_{i}=\alpha_{0}\left(8 \hbar / m_{i}^{*} \omega\right), 1 / M^{*}=1 / m_{\mathrm{e}}^{*}+$ $1 / m_{\mathrm{h}}^{*}, B_{n^{\prime} n}^{i}=\left[A_{n^{\prime}}^{i} /\left(A_{n}^{i}-A_{n^{\prime}}^{i}\right)\right] \ln \left[A_{n}^{i}\left(1+A_{n^{\prime}}^{i}\right) / A_{n^{\prime}}^{i}\left(1+A_{n}^{i}\right)\right]$ with $A_{n}^{i}=\exp \left[\left(\varepsilon_{n}^{i}-E_{\mathrm{F}}\right) / k_{\mathrm{B}} T\right]$, and $F_{n n^{\prime}}^{i j}=f\left(x_{j}^{-}\right)\left[1-f\left(x_{i}^{+}\right)\right]$with $x_{i}^{ \pm}=\left(m_{\mathrm{e}}^{*} \varepsilon_{n}+m_{\mathrm{h}}^{*} \varepsilon_{n^{\prime}} \pm m_{i}^{*} \hbar \omega\right) /\left(m_{\mathrm{e}}^{*}+m_{\mathrm{h}}^{*}\right)$. Furthermore, for the case of intra-layer scattering, in equation (6) we have taken $\delta(x) \rightarrow\left(\Gamma_{i} / \pi\right) /\left(x^{2}+\Gamma_{i}^{2}\right)$ with $\Gamma_{i}$ being the broadening of the scattering state.

\section{Numerical results and discussions}

In the present study, we consider a typical InAs/GaSb QW in which the type II and brokengap structure can be achieved experimentally [7]. The widths of the InAs and GaSb layers are taken respectively as $L_{\mathrm{InAs}}=17 \mathrm{~nm}$ and $L_{\mathrm{GaSb}}=5 \mathrm{~nm}$. In such a structure, the electron 


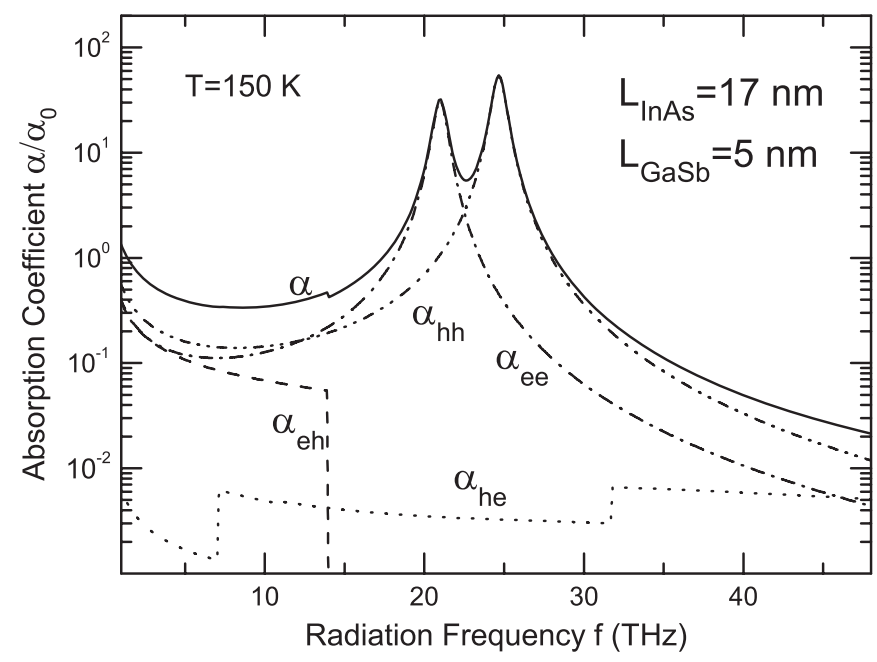

Figure 2. Contribution from different transition channels to optical absorption coefficient $\alpha$ at fixed temperature $T$ and QW widths $L_{\mathrm{InAs}}$ and $L_{\mathrm{GaSb}}$. Here $\alpha_{i j}$ refers to the absorption coefficient induced by transition from layer $i$ to layer $j$ and $\alpha_{0}=e^{2} /\left(\hbar \sqrt{\kappa} \epsilon_{0} C\right)$ is a normalization coefficient.

and hole density along with the quantum mobilities for electrons and holes can be measured via magnetotransport experiments [7]. Using the material parameters for InAs and GaSb and the corresponding heterostructure, we calculate the electronic subband structure by solving the Schrödinger equation and Poisson equation self-consistently [8]. The results obtained from this calculation are: electron density $n_{\mathrm{e}}=1.14 \times 10^{12} \mathrm{~cm}^{-2}$, hole density $n_{\mathrm{h}}=3.10 \times 10^{11} \mathrm{~cm}^{-2}$, $\varepsilon_{1}^{\mathrm{e}}=32.0 \mathrm{meV}, \varepsilon_{2}^{\mathrm{e}}=119.9 \mathrm{meV}, \varepsilon_{1}^{\mathrm{h}}=106.3 \mathrm{meV}$ and $\varepsilon_{2}^{\mathrm{h}}=4.2$ measured from the bottom of the conduction band in the InAs layer. In such a structure, only the lowest electron-subband and the highest hole-subband are occupied respectively by electrons and holes [7, 8]. The details of the electronic subband structure in this sample system can be found in [8]. In the calculation, the temperature-dependent Fermi-energy (or chemical potential) is determined by the condition of charge number conservation, namely by solving $n_{\mathrm{e}}=g_{\mathrm{s}} \sum_{n, \mathbf{k}} f\left(E_{i}^{\mathrm{e}}(k)\right)$ with $g_{\mathrm{s}}=2$ counting for spin degeneracy and $f(x)$ being the Fermi-Dirac function. For calculating $\alpha_{i j}$, we take two lowest electron-subbands and two highest hole-subbands (i.e. channels 1-7 shown in figure 1 are all included) and take $\Gamma_{\mathrm{e}}=\Gamma_{\mathrm{h}}=1 \mathrm{meV}$.

In figure 2, we show contributions from different transition channels to optical absorption in an InAs/GaSb QW with $L_{\mathrm{InAs}}=17 \mathrm{~nm}$ and $L_{\mathrm{GaSb}}=5 \mathrm{~nm}$. As can be seen, the major contribution to the absorption coefficient comes from inter-subband transition within the same well layer (i.e. process 2 for holes in the GaSb layer and process 5 for electrons in the InAs layer, shown in figure 1). Because the subband-energy separations for electrons and holes in the InAs and GaSb layers are different, two absorption peaks can then be observed respectively at $\hbar \omega \sim \varepsilon_{1}^{\mathrm{e}}-\varepsilon_{0}^{\mathrm{e}}$ and $\hbar \omega \sim \varepsilon_{0}^{\mathrm{h}}-\varepsilon_{1}^{\mathrm{h}}$. Because $\varepsilon_{1}^{\mathrm{e}}-\varepsilon_{0}^{\mathrm{e}} \sim 88 \mathrm{meV}$ and $\varepsilon_{0}^{\mathrm{h}}-\varepsilon_{1}^{\mathrm{h}} \sim 102 \mathrm{meV}$, two absorption peaks can be observed in the mid-infrared bandwidth. When $L_{\text {InAs }}=17 \mathrm{~nm}$ and $L_{\mathrm{GaSb}}=5 \mathrm{~nm}$, the electron and hole distribution along the growth-direction is highly localized and the overlap of the electron and hole wavefunctions at the interface is relatively small [8]. The optical absorption induced by inter-band or inter-layer transitions is therefore relatively weak. In a very recent theoretical work [4], we have examined the strength of electronic transition or charge transfer between InAs and GaSb layers caused by linearly polarized radiation fields. It was found that in an InAs/GaSb QW with $L_{\mathrm{InAs}}=17 \mathrm{~nm}$ and $L_{\mathrm{GaSb}}=5 \mathrm{~nm}$, 


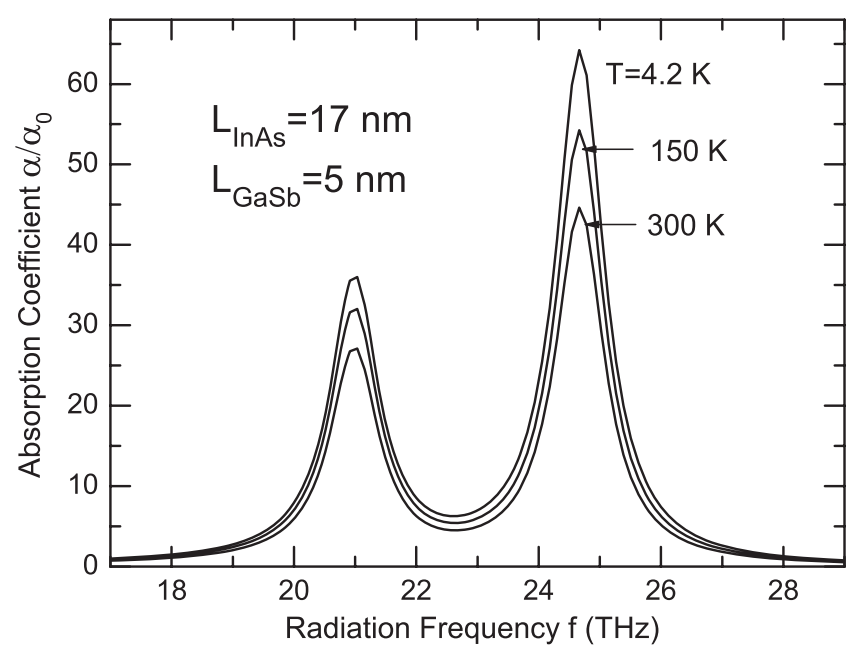

Figure 3. Temperature dependence of the optical absorption spectrum at fixed InAs and GaSb layer thicknesses.

the inter-layer scattering rate induced by electron/hole interactions with sub-THz fields is about $10^{11} \mathrm{~s}^{-1}$ (or $0.1 \mathrm{THz}$ ). This scattering rate is even smaller for electron/hole interactions with mid-infrared radiations. The numerical results show that for inter-subband scattering induced by electron/hole interactions with mid-infrared fields within the InAs/GaSb layer, the scattering rate can reach up to $10^{12} \mathrm{~s}^{-1}$ (or THz). Thus, the strength of inter-subband or intra-layer optical absorption is much stronger than that of inter-band or inter-layer absorption.

In figure 3, we show the temperature dependence of the two absorption peaks induced by inter-subband transition for electrons and holes within, respectively, the InAs and GaSb layers. We find that over a wide temperature range, two sharp absorption peaks can be observed and the intensity of the photon absorption decreases rather weakly with increasing temperature up to room-temperature. In an InAs/GaSb-based type II and broken-gap QW, the confinement potentials for electrons (about $2 \mathrm{eV}$ ) and holes (about $0.6 \mathrm{eV}$ ) are much stronger than those in III-V based QWs. With relatively narrow widths of the InAs $\left(L_{\mathrm{InAs}}=17 \mathrm{~nm}\right)$ and $\mathrm{GaSb}$ $\left(L_{\mathrm{GaSb}}=5 \mathrm{~nm}\right)$ well layers, the subband-energy separations for electron and hole layers are relatively large. Due to the large energy spacing between $\varepsilon_{1}^{\mathrm{e}}$ and $\varepsilon_{0}^{\mathrm{e}}$ and between $\varepsilon_{0}^{\mathrm{h}}$ and $\varepsilon_{1}^{\mathrm{h}}$, the thermal occupation of electrons and holes to the first excited subbands is very weak up to room-temperature. Together with the fact that the optical transition energies for exciting electrons and holes in different subbands are larger than the thermal energy $k_{\mathrm{B}} T$, the strength of optical absorption in an InAs/GaSb-based type II and broken-gap QW depends weakly on temperature up to room-temperature.

It is known that with increasing the width of the well layer, $\left|\varepsilon_{1}^{j}-\varepsilon_{0}^{j}\right|$ decreases. Thus, in principle we can tune the frequencies of the two-colour absorption by varying the width of the InAs and/or GaSb well layers. However, the change of the InAs and/or GaSb thickness can induce changes of the hybridization of the subband structure and of the occupancy of carriers to different subbands. In the present study, we limit ourselves to an InAs/GaSb-based type II and broken-gap QW system realized successfully in very recent experiments [7]. When $L_{\mathrm{InAs}}=17 \mathrm{~nm}$ and $L_{\mathrm{GaSb}}=5 \mathrm{~nm}$, not only a type II and broken-gap QW structure can be achieved but also the effect of hybridization of electron and hole subband structures can be largely reduced. Therefore, this structure can be used as a two-colour optoelectronic device. 
As pointed out on the basis of the $\mathbf{k} \cdot \mathbf{p}$ band-structure calculations [6], the hybridization of the electron and hole dispersions in InAs/GaSb QWs depends strongly on the widths of the InAs and GaSb layers. The results obtained from self-consistent calculations [8] indicate that the achievement of the type II and broken gap QW structure depends also on other sample parameters such as the GaSb cap layer and the presence of intrinsic defects in the AlSb barrier layer. Therefore, in the present study we do not attempt to examine the dependence of the two-colour optical absorption on widths of the InAs and GaSb well layers.

\section{Conclusions}

In this work, we have demonstrated theoretically that in an InAs/GaSb-based type II and broken-gap QW with $L_{\mathrm{InAs}}=17 \mathrm{~nm}$ and $L_{\mathrm{GaSb}}=5 \mathrm{~nm}$, optical absorption from interband or inter-layer transition events is weak. Two sharp absorption peaks can be observed in the mid-infrared bandwidth due to inter-subband or intra-layer transitions for electrons/holes within the InAs/GaSb layers. The strength of this kind of two-colour absorption depends rather weakly on temperature up to room-temperature. It is known that two-wavelengths allow a precise determination of emissivity and temperature. In particular, two colours in the infrared bandwidth have important applications in, for example, the third generation of infrared photodetectors [12]. The results obtained from this study indicate that InAs/GaSb-based type II and broken-gap QW systems can be used as mid-infrared two-colour sensors working at relatively high temperatures. We hope these theoretical findings and predictions will be verified experimentally.

\section{Acknowledgments}

This work was supported by the Australia Research Council, NSF of China (Grant No. 10374091) and the Knowledge Innovation Program of Chinese Academy of Sciences. Discussions with P A Folkes (ARL) and G Gumbs (Hunter College) are gratefully acknowledged.

\section{References}

[1] See, e.g. Bastard G 1992 Wave Mechanics Applied to Semiconductor Heterostructures (Paris: Monographies de Physique) p 237

[2] Altarelli M 1983 Phys. Rev. B 28842

[3] Munekata H, Maan J C, Chang L L and Esaki L 1987 J. Vac. Sci. Technol. B 5809

[4] Wei X F, Xu W and Zeng Z 2007 J. Phys.: Condens. Matter 19026216

[5] See, e.g. Lakrimi M, Khym S, Nicholas R J, Symons D M, Peeters F M, Mason N J and Walker P J 1997 Phys. Rev. Lett. 793034

[6] See, e.g. Zakharova A, Yen S T and Chao K A 2002 Phys. Rev. B 66085312

[7] Folkes P A, Gumbs G, Xu W and Taysing-Lara M 2006 Appl. Phys. Lett. 89202113

[8] Xu W, Folkes P A and Gumbs G 2007 J. Appl. Phys. 102033703

[9] Xu W, Peeters F M and Devreese J T 1992 Phys. Rev. B 467571

[10] Xu W 2006 Appl. Phys. Lett. 89171107

[11] Lei X L and Liu S Y 2000 J. Phys.: Condens. Matter 124655

[12] See, e.g. Norton P 2006 Opto-Electron. Rev. 141 